\title{
Predictive role of intraoperative clinicopathological features of the central compartment in estimating lymph nodes metastasis status
}

\author{
Rong-Hao Sun ${ }^{1,2 \#}$, Chao Li $^{1}$, Yu-Qiu Zhou ${ }^{1 \#}$, Yong-Cong Cai ${ }^{1}$, Chun-Yan Shui ${ }^{1}$, Wei Liu $^{3}$, Xu Wang ${ }^{4}$, \\ Din-Fen Zeng ${ }^{1}$, Jian Jiang ${ }^{1}$, Jing-Qiang $\mathrm{Zhu}^{2}$
}

${ }^{1}$ Department of Head and Neck Surgery, Sichuan Cancer Hospital \& Institute, Sichuan Cancer Center, School of Medicine, University of Electronic Science and Technology of China, Chengdu 610041, China; ${ }^{2}$ Department of Thyroid Surgery, West China Hospital, Sichuan University, Chengdu 610000, China; ${ }^{3}$ Department of Otolaryngology Head and Neck Surgery, The Affiliated Hospital of Southwest Medical University, Luzhou 646000, China; ${ }^{4}$ Chengdu Medical College, Chengdu 610083, China

Contributions: (I) Conception and design: R Sun; (II) Administrative support: C Li, JQ Zhu, YC Cai; (III) Provision of study materials or patients: Y Zhou, C Shui, W Liu, X Wang; (IV) Collection and assembly of data: R Sun, Y Zhou; (V) Data analysis and interpretation: R Sun, C Li; (VI) Manuscript writing: All authors; (VII) Final approval of manuscript: All authors.

\#These authors contributed equally to this work

Correspondence to: Chao Li. Department of Head and Neck Surgery, Sichuan Cancer Hospital \& Institute, Sichuan Cancer Center, School of Medicine, University of Electronic Science and Technology of China, Chengdu 610041, China. Email: headneck@qq.com; Jing-Qiang Zhu. Department of Thyroid Surgery, West China Hospital, Sichuan University, Chengdu 610041, China. Email: zjq-wkys@163.com

Background: To explore the feasibility of immediate assessment, which focuses on clinicopathological characteristics of central lymph nodes (LNs) during operation. Moreover, to analyze the predictive effect of various evaluated indicators on the nature, quantities, and ratios of central lymph node metastasis (LNM) in papillary thyroid carcinoma (PTC), to provide the basis for precise individualized central lymph node dissection (LND).

Methods: According to the inclusion and exclusion criteria, 1,271 PTC patients were selected in this cohort study. In the study, the clinical and pathological characteristics of the central LNs were evaluated by the treatment groups during the operation, which had a similar therapeutic experience. The parameters including the texture, volume, maximum/vertical meridian, extracapsular infiltration, adhesion or fusion, and nano-carbon staining status of the central LNs were collected. According to the pathological results after the operation, the nature, quantities, and rate of LNM in the central compartment were counted. The relationship between these parameters and metastatic nature, quantities, and ratios was analyzed and compared.

Results: Univariate analysis showed that when the larger size of LNs (especially the maximum meridian $>0.9 \mathrm{~cm}$ ), extracapsular infiltration, adhesion and fusion being found, A higher possibility of LNM in the central compartment $(\mathrm{P}<0.05)$, higher number and ratio of metastasis $(\mathrm{P}<0.05)$ might be existed. Moreover, more than two positive LNs were more likely to appear. Maximum/vertical meridian <2 and texture hardness could not indicate metastasis $(\mathrm{P}>0.05)$ and higher metastasis ratio $(\mathrm{P}>0.05)$, but could only be used as a reference for the existence of metastasis $(\mathrm{P}<0.05)$. The number of metastatic LNs dissected by carbon nanoparticles during operation could be increased $(\mathrm{P}<0.05)$. However, it has no predictive effect on the nature and rate of LNM $(\mathrm{P}>0.05)$. Multivariate analysis showed that larger central LNs, the maximum meridian $>0.9 \mathrm{~cm}$, extracapsular infiltration, adhesion, and fusion were independent prognostic factors for central LNM $(\mathrm{P}<0.05)$, which could be used as a predictor of the properties of central LNs during operation. At the same time, larger LNs, extracapsular infiltration, adhesion and fusion, and nano-carbon black staining were independent predictors of LNM in the central compartment, which are more than two $(\mathrm{P}<0.05)$.

Conclusions: It is practical and feasible to evaluate the clinicopathological features of central LNs immediately during the operation. Intraoperative assessment of central LNs volume, capsular infiltration, maximum/vertical meridian, carbon nano tracking, and adhesion and fusion has predictive effects differently on the nature, quantities, and ratios of central LNM. In order to make an early prediction and advance 
judgment, surgeons should pay more attention to evaluate clinicopathological features of central LNs during operation, which is conducive to the proper implementation of LND in the central compartment.

Keywords: Differentiated thyroid carcinoma; clinicopathological features; central lymph node metastasis (LNM); central lymph node dissection (central LND)

Submitted Jul 01, 2019. Accepted for publication Jul 17, 2019.

doi: $10.21037 / \mathrm{atm} .2019 .08 .01$

View this article at: http://dx.doi.org/10.21037/atm.2019.08.01

\section{Introduction}

The incidence of differentiated thyroid cancer (DTC) is increasing rapidly year by year. The discussion of standardized diagnosis and treatment of DTC has been a hot topic in academic exchanges all over the world in recent years. Because papillary thyroid carcinoma (PTC) is the primary type of DTC, and neck lymph node metastasis (LNM) is the most common and essential way of PTC metastasis. Thus, the prevalence of LNM is high in all neck compartments, including the central compartment, which is the first step of lymph nodes (LNs) to metastasis. However, the metastasis of LNs in the central compartment is usually microscopic and not detectable, which is difficult to evaluate before operation accurately.

Moreover, because the anatomical relationship of this region is unique, operative procedures often cause or aggravate recurrent laryngeal nerve paralysis and hypocalcemia after the operation.

Furthermore, the unproven benefit in terms of recurrence and mortality are significant concerns (1). For this reason above, there is still controversy about prophylactic central node dissection (CND) for Initially treated DTC patients which by definition is performed when preoperative suspected and intraoperative LNM are not present (cN0) (2). It is also the focus of debates among scholars in this professional field.

In order to better realize the standardized management of the central compartment and to improve the accuracy of medical decision-making, surgeons should try their best to make patients benefit from correct medical treatment. In recent years, a large number of studies have been carried out on the relationship between clinicopathological features and LNM of DTC. Comprehensive assessment of primary lesions has gradually been recognized as the predictor of LNs status in the central compartment, and many factors are used as high-risk factors in national guidelines to assist the choice of CND. However, in the long-term clinical practice, relying solely on the primary lesion to predict the metastasis of the central LNs is not accurate enough. So a more accurately and directly evaluation method needs to be improved urgently. Since the central compartment is often well exposed after the resection of the primary lesion, then surgeons can directly evaluate the characteristics and status of the central LNs by visual, tactile and other assistant means during the operation (Figure 1A). To combine the characteristics of the primary tumor with central LNs during operation may be a better predictive mean to evaluate the LNM in the central compartment. In order to clarify the above viewpoints, some variables are selected in this study which are strictly related to the judgment of the metastasis of LNs during operation and concerned by surgeons. This research retrospectively analyzed the impact of these variables on the nature, number, and rate of LNM in the central compartment.

\section{Methods}

\section{Patients}

This retrospective analysis enrolled 1,271 DTC patients who underwent initial operation in head and neck surgeons at Cancer Hospital Affiliated to Medical College of University of Electronic Science and Technology/Sichuan Cancer Hospital from January 2015 to December 2018. All patients were diagnosed through postoperative pathology. Pathological staging was between T1N0M0 and T4N1M0, which all were papillary. Before treatment, all patients gave informed consent, which was approved by the Sichuan Cancer Hospital Institutional Ethics Committee. The type of LNM was confirmed the same as the primary tumor. Patients with a history of prior treatment for head and neck cancer or other thyroid malignancies were excluded from this study. Patients whose cases combined with highly aggressive subtypes, distant systemic metastasis, lack of preoperative imaging studies and incomplete data were also 

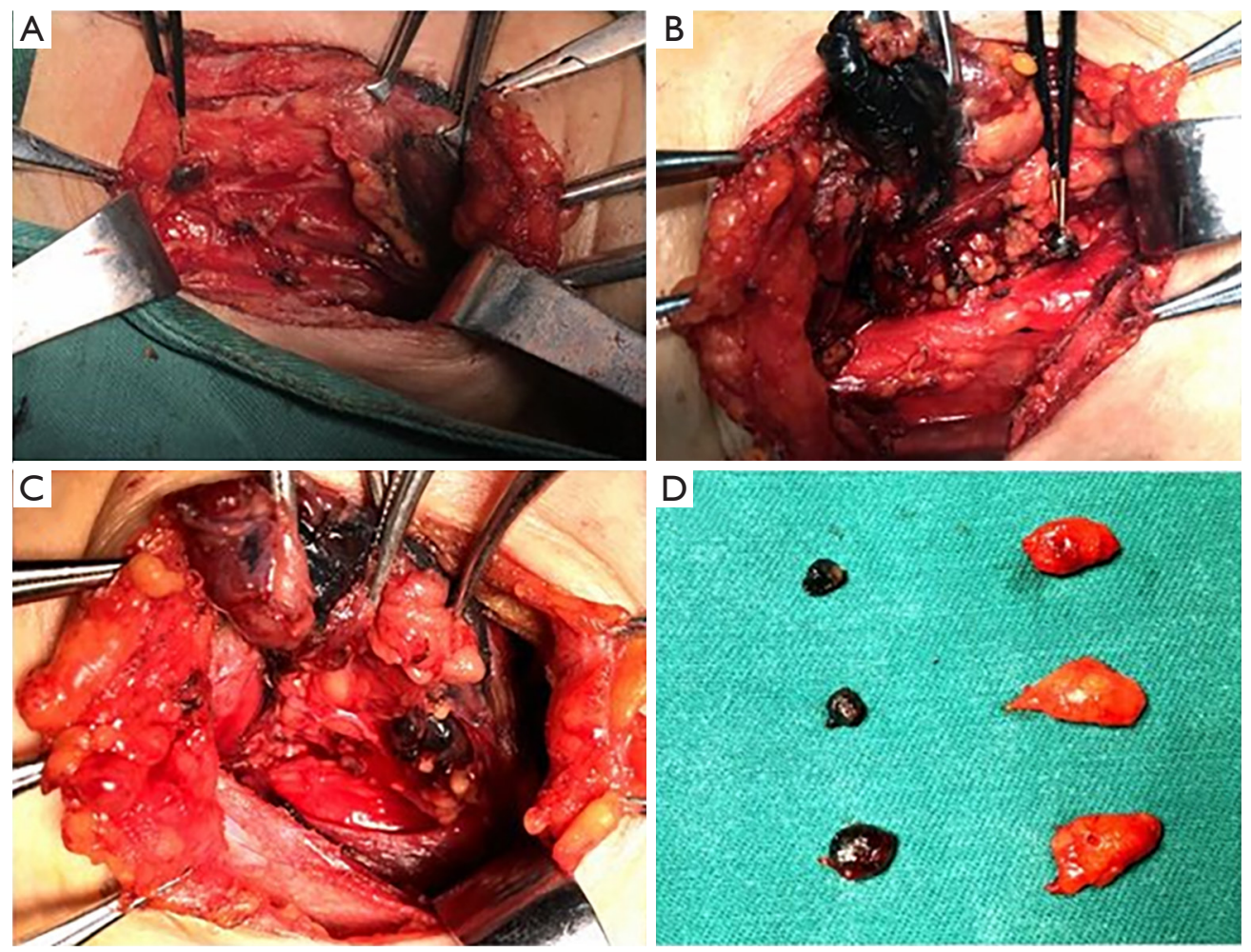

Figure 1 Effective exposure of central compartment and comprehensive assessment of central LN status during operation. (A) Thyroid gland lobes were lifted to expose the central compartment, and central LNs were seen; (B) central LNs with nano-carbon black staining, aspect ratio <2, and no adhesion and fusion; (C) central LNs with nano-carbon black staining, aspect ratio <2, adhesion and fusion; (D) central LNs of nano-carbon black staining, aspect ratio $<2$, hard texture were compared with nano-carbon being not black dyed, aspect ratio $>2$ and soft texture. LN, lymph node.

excluded.

\section{Surgical procedure}

All patients underwent open surgery, which presented total thyroidectomy or lobectomy with isthmusectomy firstly. Moreover, then selective dissection of the bilateral or unilateral central compartment was used for all these cases, which may be combined with lateral neck dissection in some cases. The central compartment of the thyroid gland is defined as level VI, which includes the pretracheal, paratracheal, and prelaryngeal LNs. The lateral compartment is defined as level II-V. Before lymph node dissection (LND), carbon nanoparticles were injected routinely into the thyroid to help with $\mathrm{LN}$ tracing and negative parathyroid imaging (Figure 1B,C). In these cases, elective lateral LND was performed because suspicious LNs were detected through imaging examinations or palpation. The thymus was preserved through separation from the
LND, and the parathyroid and recurrent laryngeal nerves were also identified and preserved. Parathyroid glands that could not be preserved in situ were auto-transplanted into the ipsilateral sternocleidomastoid muscle. All specimens were sent to the pathology department for intraoperative frozen section and postoperative paraffin section examination.

\section{Clinicopathologic variables of central LNs}

In the retrospective analysis, we first collected the necessary clinical information of 1,271 patients (including pathology, gender, age and so on). Then the metastasis of LNs in the central compartment (including the nature of metastasis, the number of metastases, and the ratio of metastasis) was counted. At the same time, the intraoperative findings (including the texture, volume, ratio of maximum diameter to vertical meridian, extracapsular infiltration, adhesion or fusion, and nano-carbon staining) of the central LNs 
were recorded in detail. In the intraoperative evaluation of texture, surgeons in this study used palpation to classify it as hard and soft, and measured the maximum meridian of LNs as the size criteria and divided them into $<0.9$ and $<0.9 \mathrm{~cm}$ groups according to the literature and our statistical analysis results. In analyzing the ratio of the maximum diameter to the vertical meridian, the aspect ratio was chosen as the criterion of benign and malignant LNs, which is generally applicable in color Doppler ultrasonography. The surgeon judged extracapsular infiltration, adhesion or fusion, and nanocarbon staining during the operation. The number and rate of LNM in the central region were based on the pathological results after operation. In the process of analyzing the number of metastasis, not only quantitative statistics were carried out, but also classified statistical analysis was performed that the number of metastasis was divided into $<2$ and $>2$ according to the bound of the mean of metastasis. All data were collected and collated by two full-time staff from Head and Neck Surgery Center of Sichuan Cancer Hospital.

\section{Statistical analysis}

A sample size calculation was performed to provide the number of patients necessary for evaluation. Clinicopathologic characteristics related to LNM were evaluated using univariate and multivariable analysis. Continuous variables were compared using the Student's $t$-test. Bivariate correlate analysis was selected to assess the interaction between two continuous variables. Categorical data were analyzed using the chi-square test or Fisher's exact test. Logistic regression was used for multivariable analysis. All statistical analysis was performed using a statistical package (SPSS, version 17.0; Chicago, IL, USA), and a $\mathrm{P}$ value of less than 0.05 was considered statistically significant.

\section{Results}

\section{Demographic and clinicopathologic characteristics of central LNs}

The final study cohort of 1,271 patients consisted of 940 (74.0\%) females and $331(26.0 \%)$ males. The sex ratio (male/female) was $7 / 20$. The mean age of the patients was $42.7 \pm 12.8$ (range, 7-86) years. Among them, 1,047 (82.4\%) were younger than 55 years old, and $224(17.6 \%)$ were older than 55 years old. All the types of the tumor were papillary. The average number of LND in the central compartment was $6.89 \pm 5.33$ in each patient. The average metastasis rate of central LNs was $29.7 \pm 34.0$. There were 704 (55.4\%) positive LNs in the central compartment, and 567 (44.6\%) were negative. Nine hundred and eighteen $(72.2 \%)$ had less than two metastases, and $353(27.8 \%)$ had more than two metastases. The mean maximum diameter of dissected central LNs was $0.90( \pm 0.46 \mathrm{~cm})$. Regarding $0.9 \mathrm{~cm}$ as the cut-off value, there was $673(53.0 \%)$ patients $<0.9 \mathrm{~cm}$ and $598(47.0 \%)$ patients $>0.9 \mathrm{~cm}$. One hundred and sixtytwo $(12.7 \%)$ cases had extracapsular LNs infiltration, and $1,109(87.3 \%)$ cases had no LNs infiltration. The central LNs of 507 (39.9\%) patients were evaluated as adhesions or fusion during operation, while $764(60.1 \%)$ patients were negative. The ratio of maximum/vertical diameter $<2$ was $1,048(82.5 \%)$ and $\geq 2$ was $223(17.5 \%)$. Six hundred thirtyseven cases $(50.1 \%)$ had hard LNs, and 634 cases (49.9\%) were soft LNs. Nine hundred seventy-five $(76.7 \%)$ of the patients were blackened or uniformly stained with nanocarbon. Two hundred ninety-six $(23.3 \%)$ patients were not or poorly stained during the operation. All the data above are shown in Table 1.

\section{Comparison central LNM status, number, and ratio according to clinicopathologic features of intraoperative evaluation in the central compartment}

Through univariate analysis, researchers in this study analyzed the influence of clinicopathological characteristics and variables in the central compartment to LNM status, several metastases, and metastasis ratio. From the results, nano-carbon staining and the ratio of maximum/vertical diameter of central LNs $<2$ had no significant effect on the metastasis of central LNs $(\mathrm{P}>0.05)$. While larger LNs (especially $>0.9 \mathrm{~cm}$ ), harder texture, extracapsular infiltration, adhesion, and fusion between LNs could increase LNM risk $(\mathrm{P}<0.05)$, which is shown in Table 2.

In comparison with the number of LNM, slightly different results were found. LN texture and maximum/ vertical diameter $<2$ could not be used as a predictor of more LNM $(\mathrm{P}>0.05)$, while carbon nanoparticles injection was helpful for dissection and detection of more metastatic LNs $(\mathrm{P}<0.05)$. Other variables such as larger LNs (especially $>0.9 \mathrm{~cm}$ ), extracapsular immersion, $\mathrm{LN}$ adhesion, and fusion are associated with more LNM, as shown in Table 3. When the number of LNM was further classified and compared $(<2$ or $>2$ ), the results were consistent, as shown in Table 2.

In predicting the positive rate of central LNM, there was no statistical difference between the texture of LNs 
Table 1 Demographic and clinicopathological characteristics of central LNs with 1,271 DTC patients

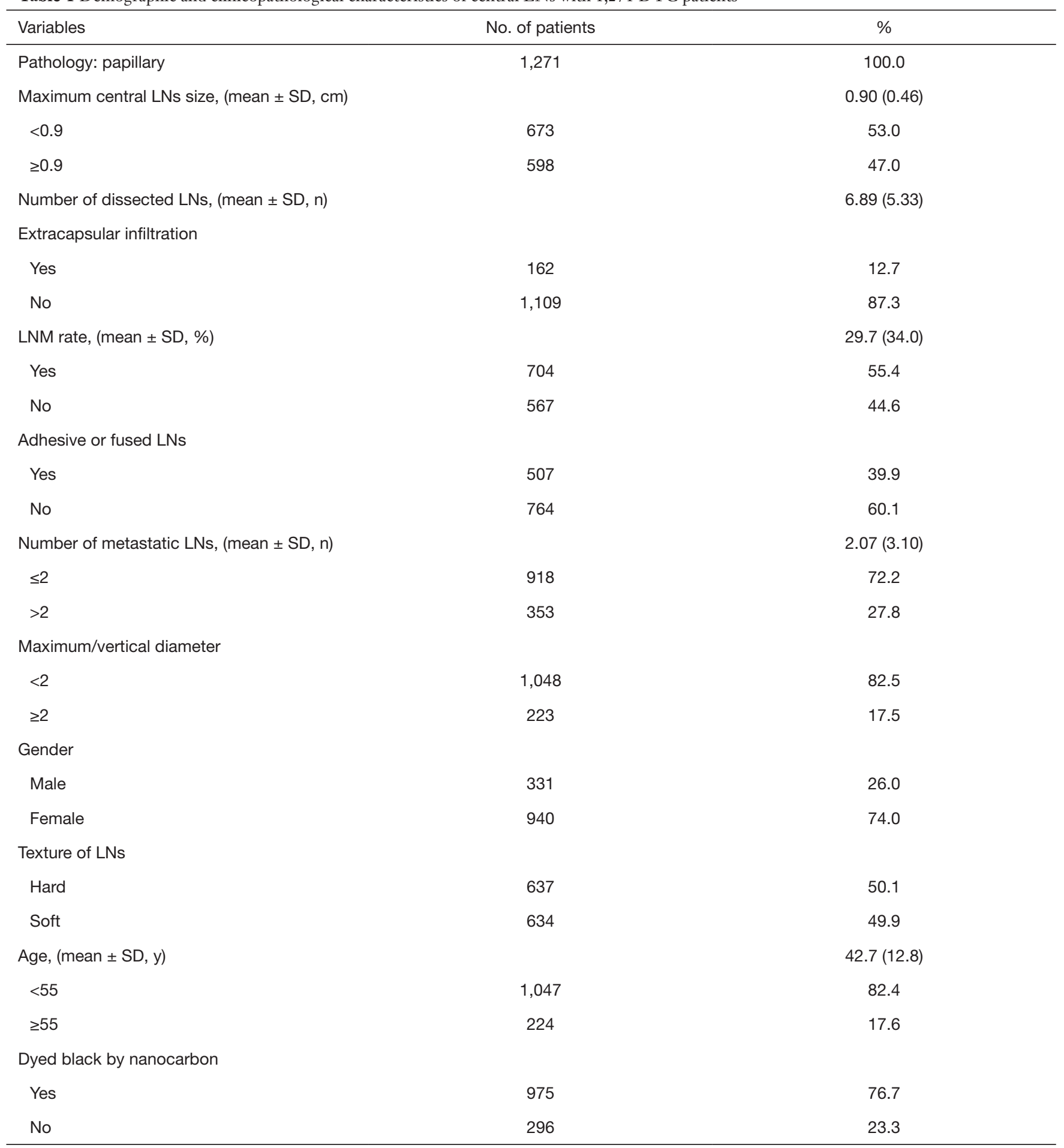

LNs, lymph nodes; LNM, lymph node metastasis; DTC, differentiated thyroid cancer; SD, standard deviation. 
Table 2 Univariate analysis of clinicopathological features and status of central LNs related to central LNM and number of central LNM from 1,271 DTC patients

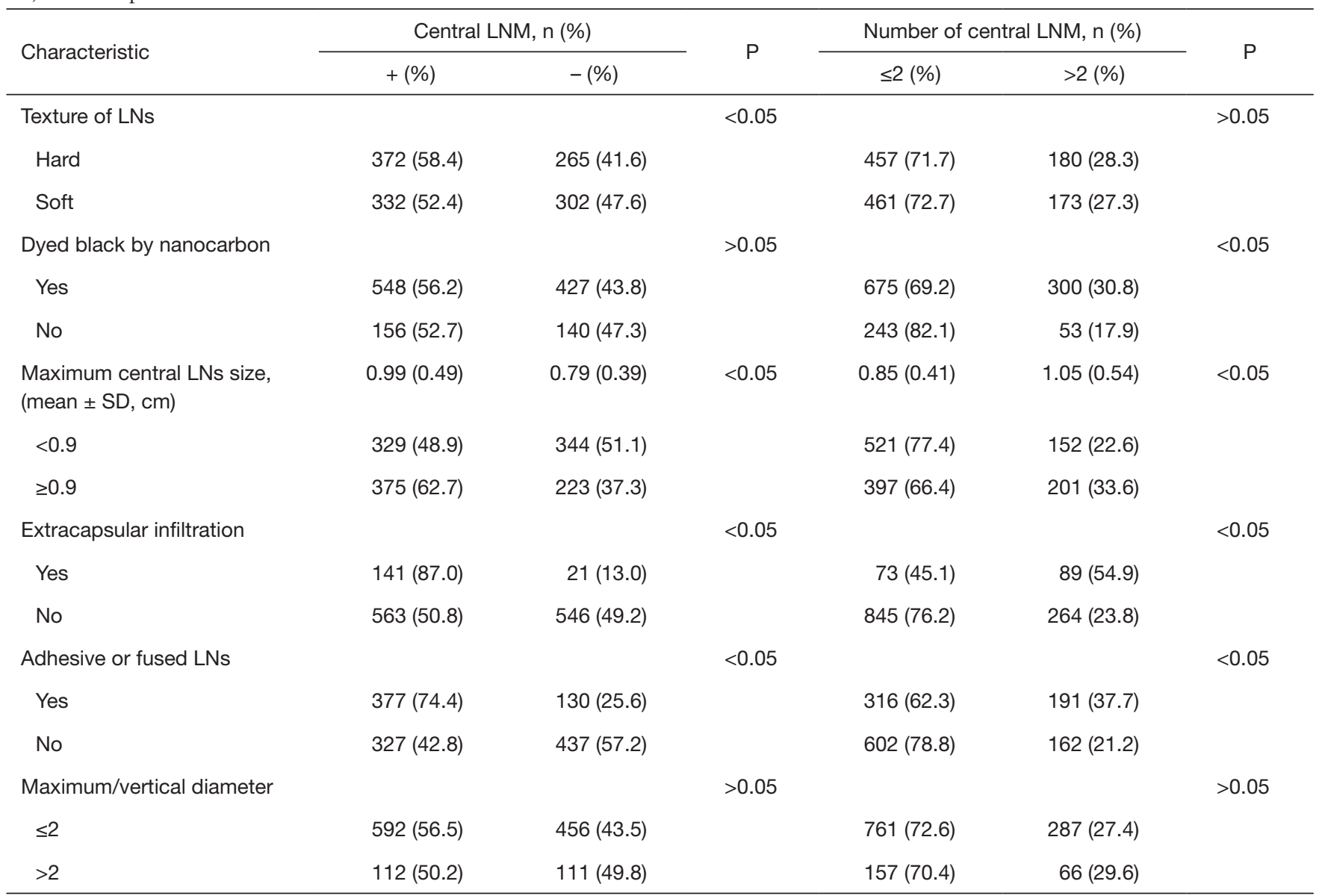

LNs, lymph nodes; LNM, lymph node metastasis; DTC, differentiated thyroid cancer; SD, standard deviation.

and the role of carbon nano tracer in this analysis $(\mathrm{P}>0.05)$. However, the volume, texture, extracapsular infiltration, adhesion, and fusion may affect the rate of LNM $(\mathrm{P}<0.05)$. In quantitative analysis, the maximum diameter of LNs was not only related to metastatic status in the central compartment but also positively related to the ratio of metastasis $(\mathrm{P}<0.05)$, as shown in Table 3.

\section{Multivariable logistic regression analysis}

For binary variables, after completing the univariate analysis, Researchers in this study further screened out the potential predictors with statistical differences in univariate analysis, and then carried out binary logistic regression analysis. The results showed that the larger LNs in the central compartment, the largest diameter of LNs $>0.9 \mathrm{~cm}$, the extracapsular invasion, adhesion, and fusion were independent prognostic factors of central LNM, which could be used as a predictor of the metastatic status in the central compartment during operation. At the same, the larger central LNs, the extracapsular infiltration, adhesion and fusion, and nano-carbon black staining were independent predictors of more than 2 LNM in the central compartment. The data are shown in Table 4.

\section{Discussion}

Surgical treatment of thyroid cancer has always been an essential part of its standardized medical process, and the extent of surgery has been widely disputed. Especially for PTC, neck LNM is not only its primary way of metastasis but also an essential factor affecting the prognosis of PTC patients. At present, there is still controversy on standardized management of neck LNs in the world. The 
Table 3 Univariate analysis of clinicopathological features and status of central LNs related to rate of central LNM and number of central LNM from 1,271 DTC patients

\begin{tabular}{|c|c|c|c|c|}
\hline Characteristics & $\begin{array}{l}\text { Rate of central LNM, } \\
\quad(\text { mean } \pm S D, \%)\end{array}$ & $\mathrm{P}$ & $\begin{array}{c}\text { Number of central LNM, } \\
(\text { mean } \pm \text { SD, } n)\end{array}$ & $P$ \\
\hline Texture of LNs & & $>0.05$ & & $>0.05$ \\
\hline Soft & $28.9(34.1)$ & & $1.96(2.98)$ & \\
\hline No & $32.8(36.8)$ & & $1.35(1.87)$ & \\
\hline Maximum central LNs size, $($ mean $\pm \mathrm{SD}, \mathrm{cm})$ & Spearman correlation & $<0.05$ & Spearman correlation & $<0.05$ \\
\hline$<0.9$ & $24.3(31.8)$ & & $1.69(2.64)$ & \\
\hline No & $26.5(32.9)$ & & $1.78(2.79)$ & \\
\hline Adhesive or fused LNs & & $<0.05$ & & $<0.05$ \\
\hline Yes & $39.6(33.9)$ & & $2.79(3.43)$ & \\
\hline No & $23.2(32.5)$ & & $1.59(2.76)$ & \\
\hline Maximum/vertical diameter & & $>0.05$ & & $>0.05$ \\
\hline$\leq 2$ & $30.6(34.3)$ & & $2.11(3.19)$ & \\
\hline$>2$ & $25.8(32.2)$ & & $1.88(2.61)$ & \\
\hline
\end{tabular}

LNs, lymph nodes; LNM, lymph node metastasis; DTC, differentiated thyroid cancer; SD, standard deviation.

Table 4 Multivariate logistic regression analysis clinicopathological features and status of central LNs for central LNM and number of central LNM

\begin{tabular}{|c|c|c|c|c|}
\hline Variables & \multicolumn{2}{|c|}{ Central LNM $( \pm)$} & \multicolumn{2}{|c|}{ Number of central LNM ( $\leq 2 />2)$} \\
\hline Extracapsular infiltration & $<0.05$ & $1.48(0.49-4.38)$ & $<0.05$ & $3.01(2.09-4.35)$ \\
\hline Maximum central LNs size, mean & $<0.05$ & $1.53(1.12-2.09)$ & $<0.05$ & $2.51(1.50-4.18)$ \\
\hline Maximum central LNs size, $(<0.9 / \geq 0.9)$ & $<0.05$ & $1.37(0.70-2.69)$ & $>0.05$ & $0.64(0.41-1.01)$ \\
\hline Adhesive or fused LNs & $<0.05$ & $0.92(0.67-1.27)$ & $<0.05$ & $2.02(1.54-2.65)$ \\
\hline Dyed black by nanocarbon & - & - & $<0.05$ & $2.15(1.52-3.03)$ \\
\hline
\end{tabular}

LNs, lymph nodes; LNM, lymph node metastasis; Cl, confidence interval

extent of primary lesion resection is considered to be more radical in the United States and other European countries. However, LND is relatively conservative, and scholars mostly hold a wait-and-see attitude. For the treatment of primary lesions, the 2015 American Thyroid Association (ATA) guidelines have changed total thyroidectomy from 
the absolute indication of the 2009 edition to the relative indication according to the results of the national study (3). However, the indication of LND is more conservative in the United States of America (4). It is advocated that neck LND should be performed after clinical manifestation and confirmation of LNM. On the contrary, the Asian countries represented by China and Japan are relatively conservative in the extent of primary lesion resection, but they are more radical when facing the problem of LND. Especially for the implementation of prophylactic CND, which is currently controversial, they advocate routine dissection $(5,6)$.

Surgeons who support prophylactic LND (especially in the central compartment) generally believe that PTC is less malignant, but there are still some patients with recurrence due to neck LNM. Performing prophylactic CND can effectively reduce the local recurrence rate (7). With time, some of the cancer cells will change and develop into refractory low-differentiated or undifferentiated cancer. Opponents argue that after LND it has a high metastasis rate confirmed by pathology after the operation, but it has little effect on the final prognosis of patients which does not affect the survival rate of patients and only shortens the disease-free survival time (8). Therefore, too active treatment may increase the incidence of complications such as recurrent laryngeal nerve injury and hypocalcemia. It also reduces the quality of life. Some researchers objectively point out that the effectiveness of prophylactic CND depends on how clinical data are interpreted and the weight distribution analysis of risk and benefit (9). Therefore, it is a challenging clinical problem that there is no reliable method for $\mathrm{cN} 0$ patients to decide whether to perform dissection and how to screen high-risk patients prone to clinical neck LNM.

Recurrence risk stratification system has been considered as an essential basis for staging and prognosis of thyroid cancer, which is different from other solid malignant tumors. In recent years, risk stratification system has been continuously improved, and many clinicopathological features of thyroid cancer patients have been introduced as predictors of recurrence risk (10). In particular, with the deepening of basic research on thyroid cancer, many molecular factors such as gene mutations for molecular diagnosis and molecular stratification have been found (11). BRAFV600E, a prominent molecule, has been introduced into the risk stratification system in ATA guidelines in 2015. Its mutation is regarded as a high-risk factor for recurrence. Some studies suggest that $B R A F$ mutation is an independent predictor of central LNM. When BRAF positive mutation occurs in PTC before the operation, CND should be routinely performed (12). Research group from Head and Neck Surgery of Sichuan Cancer Hospital has been focusing on the perfection of the risk stratification system in the early stage and researched it. The results show that the location of tumors and other clinicopathological features have an impact on neck LNM (13). Moreover, changes in serological markers such as thyroid-associated antibodies may have an impact on neck LNM and prognosis of thyroid cancer (14). Nevertheless, most of these studies focus on the clinicopathological characteristics of the primary lesion in order to predict the risk of recurrence through the evaluation of multiple indicators of the primary lesion, to guide LND and clinical decision-making.

Researchers in this study believe that for the controversial management of central LNs, it is not only necessary to fully evaluate the high-risk factors that have been included in the guidelines before the operation, but also essential to pay attention to the intraoperative evaluation of LNs in the central compartment. Because the central region can be completely exposed during the operation after resection of the primary lesion and the glands have been removed (Figure 1A). The characteristics of the LNs can predict the status of central LNs during the operation. Therefore, to envisage that the status of central LNs can be more directly predicted by some commonly used indicators in clinical work to determine whether to perform prophylactic CND. This intraoperative evaluation is considered by Surgeons to be more critical, especially for some $\mathrm{cN} 0$ patients whose neck LNs are considered harmful by preoperative clinical examination. Then Researchers in this study selected the texture, volume, extracapsular immersion, adhesion and fusion, maximum/vertical diameter, and nano-carbon black staining of LNs as evaluation indicators through the summary of work experience and literature. The relationship between these indices and the status, number, ratio of central LNM confirmed by pathology after the operation was analyzed.

According to the results of this study, the higher probability, the higher number, and rate of central LNM were confirmed by postoperative pathology when there was the larger size of central LNs, the extracapsular infiltration or adhesion, and fusion. Moreover, it is an independent predictor of central LNM. Especially when $0.9 \mathrm{~cm}$ is determined as the cutoff value of LN size by mean, and there are many significant differences which further confirms that the risk of metastasis increases when the maximum diameter exceeds $0.9 \mathrm{~cm}$. The clinician 
should attach great importance to the above indicators in intraoperative evaluation. However, hard LNs are not independent predictors of metastasis, although they may indicate the possibility of metastasis, a higher number of metastases (only quantitative analysis) and metastasis rate to some extent. It should be pointed out that aspect ratio has always been an essential index for evaluating LN benignity and malignancy in color Doppler ultrasonography. Many studies believe that when aspect ratio $>2$, the possibility of benignity is higher. While when the aspect ratio was $<2$, the development of malignancy was more likely. However, in this study, naked eye assessment of maximum/vertical meridian $<2$ did not suggest that central LNs status is more likely to be malignant which had more metastases and a higher metastasis rate (Figure 1D). The reason for this phenomenon is that the morphology of central LNs has its characteristics, which is different from that in the lateral compartment. The shape of central LNs is relatively smaller and is not elliptical (maximum/vertical meridian $>2$ ). Based on the results of this study, the maximum/vertical meridian is not useful in evaluating the central LNs. However, perhaps it is more suitable for the evaluation of lateral cervical LNs. Alternatively, in the central compartment, new cutoff values of maximum/vertical meridian should be selected out for analysis.

As a new type of LN tracer, nano-carbon has been used to assist LND in radical resection of various malignant tumors. Studies have shown that the use of nano-carbon in thyroid cancer surgery can increase the number of LND $(15,16)$. In the operation of thyroid cancer, because it can help protect parathyroid gland by negative imaging principle, our center has been using it routinely in recent years (Figure 1B,C,D). From these results, carbon nanoparticles can indeed increase the number of metastatic LNs to achieve thorough LND. However, LN blackening has no apparent selectivity, so it cannot adequately indicate the metastasis and higher rate of metastasis.

Clinicians need to pay attention not only to the nature of LNM but also to the number and rate of metastasis. Past clinical experience tells us that a higher number and rate of metastasis suggest worse prognosis. Previous studies have confirmed this theory with data. When central LNM exceeds $45 \%$, and the rate of lateral LNM exceeds $29 \%$, it indicates a higher risk of recurrence $(17,18)$. It is also believed that lateral LNM is more likely to occur when the number of central LNM is more than 2 (19). Others suggested that when the central LNM rate of $\mathrm{pN} 1 \mathrm{~b}$ patients was more than $30 \%$, it was significantly correlated with shorter overall survival and disease-specific survival (20). All these studies have suggested that the number and rate of LNM may be predictors of clinical prognosis.

Moreover, the version of 2015 ATA guideline is based on the number of LNM $(n<5)$ as the risk stratification of low-risk recurrence. Therefore, base on this study, the mean value was close to 2 by quantitative comparative analysis. When the mean value was used as the cutoff value of the metastatic number in the central compartment, several characteristics of central LNs had different predictive effects, and the high-risk features indicated a higher probability of metastasis. Clinicians should pay more attention to the number and rate of LNM than to the property of central LNs. Only in this way can we evaluate the situation of the central compartment more comprehensively. Even it can help to judge the status of the lateral compartment and the prognosis.

To sum up, it is feasible to evaluate the characteristics of central LNs during operation, and it is easy to achieve in the clinic. If finding the larger size of central LNs (especially when the maximum diameter is $>0.9 \mathrm{~cm}$ ), extracapsular infiltration, adhesion, and fusion, it may indicate the higher metastatic possibility, more significant metastatic number and a higher ratio of metastasis. Moreover, they are independent predictors of LNM in the central compartment. Maximum/vertical meridian plays a limited role in intraoperative assessment and prediction of metastasis. Carbon nanoparticles can help surgeons to increase the number of LND and the detection rate of positive LNs in the central compartment, and to improve the thoroughness of dissection. However, they cannot be used as objective indicators to predict the status of LNs. When making surgical plans for PTC patients, surgeons should not only make detailed individual stratification according to the risk stratification factors in the guidelines but also strengthen the comprehensive evaluation of the characteristics of central LNs during the operation, to obtain the correct clinical decision-making and make patients benefit from it.

\section{Acknowledgments}

None.

\section{Footnote}

Conflicts of Interest: The authors have no conflicts of interest to declare. 


\section{Page 10 of 10}

Ethical Statement: The authors are responsible for all aspects of the work in ensuring that questions related to the accuracy or integrity of any part of the work are appropriately investigated and resolved. This study was approved by the Ethics Committee of Sichuan Cancer Hospital. All patients gave informed consent.

\section{References}

1. Giordano D, Valcavi R, Thompson GB, et al. Complications of central neck dissection in patients with papillary thyroid carcinoma: results of a study on 1087 patients and review of the literature. Thyroid 2012;22:911-7.

2. Calò PG, Pisano G, Medas F, et al. Total thyroidectomy without prophylactic central neck dissection in clinically node-negative papillary thyroid cancer: is it an adequate treatment? World J Surg Oncol 2014;12:152.

3. Choi JB, Lee SG, Kim MJ, et al. Oncologic outcomes in patients with $1-\mathrm{cm}$ to $4-\mathrm{cm}$ differentiated thyroid carcinoma according to extent of thyroidectomy. Head Neck 2019;41:56-63.

4. Haugen BR, Alexander EK, Bible KC, et al. 2015 American Thyroid Association management guidelines for adult patients with thyroid nodules and differentiated thyroid cancer: the American Thyroid Association guidelines task force on thyroid nodules and differentiated thyroid cancer. Thyroid 2016;26:1-133.

5. The Japanese Society of Thyroid Surgery, The Japanese Association of Endocrine Surgeons (2010). Guidelines for management of thyroid tumor. Tokyo: Kanehara Press, 2010.

6. Editorial Group of Guidelines for the Diagnosis and Treatment of Differentiated Thyroid Cancer of the Head and Neck Cancer Professional Committee of China AntiCancer Association. Guidelines for the diagnosis and treatment of differentiated thyroid cancer. Chinese Journal of Practical Surgery 2011;31:908-13.

7. Chen L, Wu YH, Lee CH, et al. Prophylactic central neck dissection for papillary thyroid carcinoma with clinically uninvolved central neck lymph nodes: a systematic review and meta-analysis. World J Surg 2018;42:2846-57.

8. Hughes DT, Rosen JE, Evans DB, et al. Prophylactic central compartment neck dissection in papillary thyroid cancer and effect on locoregional recurrence. Ann Surg Oncol 2018;25:2526-34.

9. McHenry CR. Is prophylactic central compartment neck dissection indicated for clinically node-negative papillary
Sun et al. Intraoperative prediction of central lymph node metastasis

thyroid cancer: the answer is dependent on how the data are interpreted and the weight given to the risks and benefits. Ann Surg Oncol 2018;25:3123-4.

10. Tang T, Li J, Zheng L, et al. Risk factors of central lymph node metastasis in papillary thyroid carcinoma: a retrospective cohort study. Int J Surg 2018;54:129-32.

11. Ren H, Shen Y, Hu D, et al. Co-existence of $B R A F^{V 600 E}$ and $T E R T$ promoter mutations in papillary thyroid carcinoma is associated with tumor aggressiveness, but not with lymph node metastasis. Cancer Manag Res 2018;10:1005-13.

12. Shi C, Qin H, Ding C, et al. Association between BRAF V600E mutation and central lymph node metastasis in patients with papillary thyroid carcinoma. Zhonghua Zhong Liu Za Zhi 2015;37:123-7.

13. Sun R, Zhang H, Liu K, et al. Clinicopathologic predictive factors of cervical lymph node metastasis in differentiated thyroid cancer. Acta Otorrinolaringol Esp 2018;69:149-55.

14. Li C, Yu W, Fan J, et al. Thyroid functional parameters and correlative autoantibodies as prognostic factors for differentiated thyroid cancers. Oncotarget 2016;7:49930-8.

15. Liu Y, Li L, Yu J, et al. Carbon nanoparticle lymph node tracer improves the outcomes of surgical treatment in papillary thyroid cancer. Cancer Biomark 2018;23:227-33.

16. Xue S, Ren P, Wang P, et al. Short and long-term potential role of carbon nanoparticles in total thyroidectomy with central lymph node dissection. Sci Rep 2018;8:11936.

17. Ryu YJ, Cho JS, Yoon JH, et al. Identifying risk factors for recurrence of papillary thyroid cancer in patients who underwent modified radical neck dissection. World J Surg Oncol 2018;16:205.

18. Rubinstein JC, Dinauer C, Herrick-Reynolds K, et al. Lymph node ratio predicts recurrence in pediatric papillary thyroid cancer. J Pediatr Surg 2019;54:129-32.

19. Li Yu, Teng Changsheng, Qu Xiang, et al. The relationship between the central cervical lymph node metastasis and the lateral cervical lymph node metastasis in $\mathrm{cN} 0$ papillary thyroid cancer. Chin J Endoer Surg 2014;8:223-6.

20. Suzuki H, Koide Y, Hanai N, et al. Lymph node density in papillary thyroid carcinoma is a prognostic factor after adjusting for pathological stage. Oncotarget 2018;9:26670-8.

Cite this article as: Sun RH, Li C, Zhou YQ, Cai YC, Shui CY, Liu W, Wang X, Zeng DF, Jiang J, Zhu JQ. Predictive role of intraoperative clinicopathological features of the central compartment in estimating lymph nodes metastasis status. Ann Transl Med 2019;7(18):471. doi: 10.21037/atm.2019.08.01 